\title{
Erratum: Is the Gravitational-Wave Ringdown a Probe of the Event Horizon? [Phys. Rev. Lett. 116, 171101 (2016)]
}

Vitor Cardoso, Edgardo Franzin, and Paolo Pani

(Received 29 July 2016; published 16 August 2016)

DOI: 10.1103/PhysRevLett.117.089902

The right bottom panel of Fig. 4 in the Letter refers to the Lorentz factor $E=1.01$ and not to $E=1.5$ as reported in the Letter. For completeness, we show here the waveforms for both $E=1.01$ and $E=1.5$. All conclusions of the Letter remain unchanged.

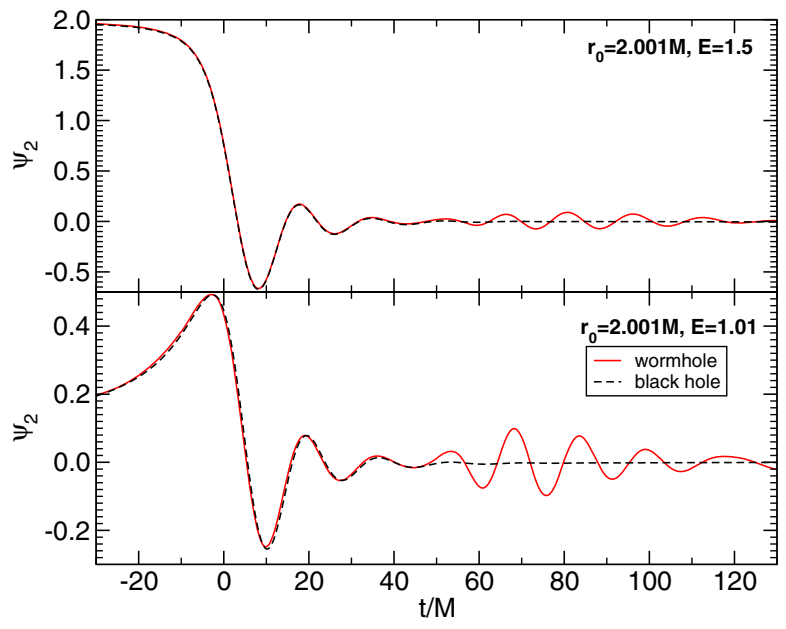

FIG. 4. Gravitational waveform for the same process presented in the right panels of Fig. 4 in the Letter, but for two choices of the Lorentz factor: $E=1.5$ (top panel) and $E=1.01$ (bottom panel). The right bottom panel of Fig. 4 in the Letter erroneously shows the $E=1.01$ case instead of the $E=1.5$ case, as reported in the caption of that figure. 